\title{
Éditorial:
}

\section{La recherche canadienne sur le vieillissement: après deux décennies de croissance, l'heure est au changement}

La publication du présent numéro de La Revue canadienne du vieillissement marque un jalon important: l'achèvement de 20 ans de publication d'un large éventail de recherches dans le domaine du vieillissement au Canada, couvrant tout le spectre de l'enquête gérontologique, des aspects biologiques du vieillissement à la gérontologie éducative en passant par les sciences de la santé, la psychologie, les sciences sociales et les politiques et pratiques sociales en relation avec les personnes âgées et vieillissantes. C'est également un jalon important pour moi personnellement, car, avec ce numéro, je termine mon mandat de quatre ans à titre de rédactrice en chef de la $R C V$, poste qu'occupera désormais Carolyn Rosenthal, de l'université McMaster. Comme ce fut le cas lorsque La Revue a célébré son dixième anniversaire (Béland, 1991), ce jalon et ce moment de transition est une occasion de réfléchir à la continuité et à l'évolution de La Revue avec le temps, aux enjeux et aux perspectives de la recherche sur le vieillissement au Canada, ainsi qu'aux spéculations sur l'avenir de ce domaine. ${ }^{1}$

\section{Les défis et les réalisations du passé}

Lorsque je suis entrée en fonction à titre de rédactrice en chef à la fin de 1996, j'ai demandé à mon prédécesseur, François Béland, de présenter en éditorial ses réflexions sur les six années qu'il avait passées à titre de rédacteur en chef de la $R C V$. Dans cet écrit, il note que si le climat de la recherche dans les années 1980 avait permis à $L a$ Revue de prospérer, l'importance des coupures budgétaires et du repli au niveau national a menacé la gérontologie dans la première moitié des années 1990 (Béland, 1997). Il souligne plus particulièrement la fin de l'initiative stratégique du CRSH sur le vieillissement de la population et la réduction des subventions fédérales et provinciales pour la recherche en gérontologie au nombre des sources d'inquiétude pour l'avenir de la gérontologie au Canada, notamment au plan du glissement vers la subvention de sujets choisis, "stratégiques," et de la disette générale de subventions à la recherche d'enquête.

Certaines des inquiétudes de M. Béland sont très bien fondées. Parmi les principaux programmes de financement nationaux, le Programme de recherche sur l'autonomie des aîné(e)s (PRAA) de Santé Canada, annoncé 
en 1988 et renouvelé pour une deuxième phase en 1993, était le seul à se consacrer encore à la recherche sur le vieillissement. L'initiative du PRAA s'est terminée en 1998. Depuis quelques années, les gouvernements provinciaux rationalisent les services de santé et réduisent le montant des subventions à la santé et à l'enseignement supérieur afin d'aider à contrôler le déficit occasionné par la baisse des paiements de transfert fédéraux (Brochu, 1995). Le milieu de la recherche connaît des conditions très variables depuis dix ans, certaines provinces consacrant peu ou pas d'argent à la recherche sur des problèmes comme le vieillissement et d'autres, comme le Québec avec le Fonds du recherche en santé du Québec (FRSQ), fonctionnant sur un modèle de centres de recherche multidisciplinaires (Chappell et al., 1999). Au cours de cette période, lorsque des subventions à la recherche axée sur le vieillissement ont été disponibles, on les a généralement accordées à des initiatives coordonnées entre plusieurs sites, comme l'Étude sur la santé et le vieillissement au Canada (ESVC), mettant l'accent sur la prévalence de la démence, et l'Étude sur l'ostéoporose au Canada.

Néanmoins, d'autres tendances inquiétantes notées en 1997 n'ont pas perduré. Confrontée à des difficultés dans sa quête de financement, la communauté des chercheurs en gérontologie a fait preuve d'innovation dans ses collaborations; lorsque c'était possible, les partenariats avec les collectivités sont devenus des éléments importants des programmes de recherche. Ces réalisations sont dignes de mention, particulièrement si on tient compte du fait que la communauté des chercheurs canadiens est toujours affligée d'une infrastructure de recherche inadéquate en gérontologie et en gériatrie.

Le financement du Réseau canadien de recherche sur le vieillissement (CARNET) de 1990 à 1995, par l'entremise du programme fédéral des Réseaux de centres d'excellence, est une des réalisations les plus remarquables de la dernière décennie. Comme le note Victor Marshall, directeur du Réseau, "[en 1990] l'idée selon laquelle les chercheurs devraient travailler en coopération active avec le secteur privé et les gouvernements à développer des recherches ayant le potentiel d'améliorer la productivité économique du Canada était très innovatrice» (CARNET, 1996).

En outre, pendant toutes les années 1990, Statistique Canada a poursuivi son engagement à créer des ensembles de données qu'elle élabore de plus en plus souvent en collaboration avec la communauté gérontologique, à qui elle donne accès à ces données; on pense notamment aux enquêtes sociales générales et aux vagues successives d'enquêtes nationales sur la santé de la population. À l'avenir, le rôle de Statistique Canada dans son travail en coopération avec la communauté des chercheurs en gérontologie sera davantage mis en valeur par l'Initiative canadienne sur les statistiques sociales (ICSS). L'ICSS vise à promouvoir la recherche et la formation sur des sujets (comme le vieillissement) qui font un plein emploi des statistiques sociales, qui facilitent l'accès à des microdonnées détaillées et 
qui maximisent l'interface entre la recherche et les politiques gouvernementales. Les neuf Centres d'accès aux données de Statistique Canada, annoncés en juillet 2000 et actuellement mis sur pied d'un bout à l'autre du pays, permettront l'analyse de données longitudinales sous forme de fichiers maîtres et la formation d'un cadre de chercheurs ayant des habiletés exceptionnelles en analyse des données. Les Enquêtes nationales sur la santé de la population sont au nombre des cinq premiers ensembles de données qui seront publiés sous cette forme par Statistique Canada; les chercheurs en gérontologie sont bien positionnés pour se prévaloir de cette occasion d'analyser des données longitudinales sur le vieillissement de la population.

En 1991, Béland a noté le rythme de croissance "spectaculaire» de La Revue depuis sa fondation en 1981. Comme on l'a vu plus haut, la deuxième décennie de La Revue s'est déroulée dans un climat beaucoup plus difficile en ce qui a trait au financement de la recherche gérontologique. Malgré cela et malgré certaines des préoccupations exprimées il y a quatre ans, le nombre de manuscrits soumis à La Revue canadienne du vieillissement s'est accru puis stabilisé au cours des quatre dernières années. Le calibre plus international de La Revue est évident dans l'augmentation du nombre d'articles publiés dont au moins l'un des auteurs est de l'extérieur du Canada, leur proportion passant de 8 pour cent des articles publiés entre 1990 et 1994 à 14 pour cent des articles publiés entre 1995 et 1999. La nature bilingue de la revue a été non seulement conservée, mais aussi améliorée depuis quelques années: si 11 pour cent des manuscrits publiés entre 1990 et 1994 étaient en français, cette proportion est passée à 17 pour cent dans la deuxième moitié de la décennie. ${ }^{2}$

L'examen des articles publiés dans la $R C V$ au cours de la dernière décennie met en relief un certain nombre de tendances dans la nature des manuscrits soumis à la rédaction. L'une d'elles est l'augmentation constante et conséquente du nombre d'articles soumis et publiés dans le domaine des sciences de la santé. Le fait a été signalé dans un éditorial récent de Connidis et collégues (2000), qui relèvent la "prédominance» des préoccupations de la recherche sur la santé en gérontologie au Canada. Ils expliquent cette situation par le fait que «les ressources humaines et financières consacrées aux soins de santé sont vastes et visibles et sont l'objet de beaucoup de débats publics» et par le "pouvoir du programme de santé» au Canada. Il reste que, bien que $L a$ Revue soit divisée en six "sections," environ le tiers de tous les manuscrits soumis sont examinés par la Section des sciences de la santé.

On constate également une augmentation du nombre d'articles soumis traitant de questions méthodologiques, des évaluations portant sur la fiabilité et la validité de la version française d'instruments d'enquête bien connus aux discussions sur la méthodologie employée dans des études d'une importance particulière, comme l'étude longitudinale sur 20 ans portant sur le vieillissement au Manitoba et l'Étude sur l'ostéoporose au 
Canada. Des questions conceptuelles et méthodologiques ont aussi été développées dans des suppléments spéciaux comme le numéro sur le vieillissement et les sciences sociales (1993) et dans des éditoriaux portant sur des sujets comme les défis de la recherche biologique fondamentale au Canada (1996). Les rédacteurs de La Revue se sont également montrés proactifs en mettant de l'avant une variété de démarches méthodologiques, par la publication de suppléments spéciaux sur les méthodes qualitatives (1993), les méthodes quantitatives (1994) et la diversité méthodologique (1995) et en contribuant au dialogue sur les applications à la recherche des données administratives comme le Minimum Data Set (2000).

Depuis dix ans, la matière des manuscrits soumis à la rédaction a également connu une certaine évolution. Les manuscrits qui traitent de la diversité de l'expérience du vieillissement, y compris au plan ethnoculturel, et du vieillissement dans les groupes "marginalisés," comme les personnes atteintes de troubles cognitifs ou d'une ou plusieurs incapacités, demeurent relativement rares, mais sont quand même plus fréquents que par le passé. Les analyses de grands ensembles de données nationaux comme les Enquêtes nationales sur la santé de la population sont de plus en plus fréquemment l'objet d'études portant sur des aspects de la santé comme les chutes et les blessures. Les articles publiés représentent aussi un éventail de disciplines plus large qu'il y a dix ans, un nombre croissant de recherches portant sur des domaines comme l'économie et la géographie. Cependant, la revue reçoit toujours une très faible proportion d'articles sur les aspects biologiques du vieillissement et le rythme de croissance des articles soumis portant sur la gérontologie éducative est demeuré lent depuis la création de cette nouvelle "section" du comité de rédaction en 1999.

Au cours de la dernière décennie en particulier, La Revue a joué un rôle de plus en plus proactif dans la promotion du dialogue sur les questions relatives aux politiques sur le vieillissement au Canada en publiant des énoncés de position de l'ACG sur les pensions (1998), les soins à domicile (1999) et les aîné(e)s et les médicaments sous ordonnance (2000). On a également vu une augmentation constante du nombre d'articles traitant de l'interface entre la recherche et les politiques et offrant des analyses de politiques critiques et rhétoriques. Ces articles vont des études individuelles traitant des modifications aux politiques provinciales sur la prestation des services de santé aux aîné(e)s à des suppléments entiers sur «Les soins de longue durée dans cinq pays» (1996), "La réécriture des politiques sociales dans une société vieillissante» (1997) et "L'établissement d'un programme politique fondé sur l'expérience pour l'autonomie des aîné(e)s» (2000). En 1997, La Revue a atteint son auditoire le plus vaste en mettant de l'avant des discussions sur les politiques dans sa publication conjointe, avec la revue Canadian Public Policy - Analyse de politiques, d'un numéro spécial sur le moyens de «Tisser des liens entre les politiques et la recherche relative au vieillissement». Ce volume, dont j'ai dirigé la publication avec 
Ellen Gee, examine l'influence de la recherche sur les choix politiques concernant le vieillissement, les étapes à franchir pour mieux lier la recherche et les politiques au bénéfice des ainé(e)s canadiens et la détermination des principaux enjeux politiques à traiter (Gee, 1997, p. i). Sa publication demeure l'accomplissement le plus satisfaisant au plan personnel au cours de mes sept ans au Comité de rédaction de la $R C V$.

\section{La promesse de l'avenir}

\section{Les Instituts de recherche en santé du Canada}

Le changement le plus important survenu dans la recherche en gérontolo. gie au Canada depuis dix ans est l'établissement au début de cette année des Instituts de recherche en santé du Canada (IRSC), en remplacement du Conseil de recherches médicales (CRM) et du Programme national de recherche et de développement en matière de santé (PNRDS) de Santé Canada. Établis par une loi du Parlement, les IRSC sont fondés sur une définition de la recherche en santé nettement plus large que dans le cadre du CRM et leur mandat comprend non seulement la recherche biomédicale et clinique, mais aussi la recherche sur les systèmes de santé et les services et la recherche sur la santé de la population.

Les IRSC redéfiniront la recherche sur le vieillissement au Canada de plusieurs façons importantes (Martin-Matthews, 2001). Premièrement, les IRSC augmenteront de façon significative les ressources financières destinées aux chercheurs en gérontologie et en gériatrie au Canada. Ils rendront accessibles aux chercheurs qui se situent à l'extérieur de ce qu'on a traditionnellement défini comme la "recherche médicale» un éventail de ressources humaines et de bourses de carrière dans le domaine de la santé autrefois réservées aux chercheurs des programmes associés aux écoles de médecine. Dans la ronde initiale d'attribution des Bourses de carrière en santé au printemps 2000 , les chercheurs en vieillissement ont été bien représentés parmi les récipiendaires de bourses offertes aux boursiers de recherches post-doctorales et aux chercheurs en début, en milieu et en fin de carrière. Les domaines de recherche subventionnés dans ces bourses de carrière comprennent des sujets qui débordent largement du mandat "traditionnel» du CRM: par exemple, la relation entre le bien-être émotionnel et l'incidence et la guérison des maladies chroniques chez les canadiens les plus âgés; la relation entre le remariage et le bien-être à la fin de la vie; les soins personnels, informels et officiels en relation avec les maladies et les incapacités chroniques au milieu et à la fin de la vie; l'informatique de la santé pour une société vieillissante; les croyances et les pratiques en matière de santé dans le contexte du vieillissement de la population; la santé des femmes âgées; la santé mentale des personnes âgées; et la santé, les soins de santé et la répartition du revenu sur la durée de la vie. Des subventions des IRSC ont déjà été offertes pour la recherche multidisciplinaire sur les implications sociales et interpersonnelles et sur la compréhen- 
sion de la démence, ainsi que sur les meilleures pratiques en matière de santé chez les personnes d'âge moyen.

Les IRSC entendent transformer l'infrastructure de la recherche sur le vieillissement au Canada par la création d'un institut national du vieillissement, l'un des 13 instituts "virtuels» qui seront établis d'ici quelques mois. L'Institut du vieillissement offrira quelque chose que nous n'avons jamais eue au Canada: "un point central national qui synthétisera ... les efforts de recherche [sur le vieillissement] et agira comme catalyseur pour la recherche [sur le vieillissement]» (Chappell et al., 1999).

La création au sein des IRSC d'un institut consacré particulièrement à la recherche sur le vieillissement est le résultat de la participation active et des commentaires de la communauté gérontologique canadienne. Cet institut soutiendra la recherche visant à promouvoir le vieillissement en santé et traitant des causes, de la prévention, du dépistage, du diagnostic, du traitement, des systèmes de soutien et des traitements palliatifs d'un large éventail de maladies associées au vieillissement. La gamme diversifiée des sujets de recherche potentiellement admissibles aux subventions des IRSC et la multidisciplinarité inhérente au mandat de l'Institut du vieillissement traduisent une vision de la recherche gériatrique et gérontologique qui est au centre de la mission et de l'attention de La Revue canadienne du vieillissement depuis sa fondation il y a 20 ans.

Il reste à voir comment les IRSC transformeront la recherche sur le vieillissement au Canada. Au moment d'écrire ces lignes, le directeur scientifique de l'Institut du vieillissement et les membres de son conseil consultatif n'avaient pas encore été nommés. Bien que la création de l'Institut du vieillissement ait généré des attentes élevées au sein de la communauté nationale des chercheurs, on exprime aussi des réserves. Certains doutent que l'IRSC puisse tenir ses promesses et que la communauté élargie des chercheurs "non médicaux» du domaine de la santé bénéficient de cette initiative. On craint également que des recherches valables en gérontologie qui ne cadrent pas précisément avec la rubrique ou même avec la définition élargie de la recherche en santé n'aient d'autre choix que de tenter d'obtenir une part du financement plus limité disponible dans les autres conseils de subvention et agences de financement. L'avenir nous dira si ces craintes sont fondées.

\section{Conclusion}

Les deux dernières décennies ont vu des changements significatifs dans le climat de la recherche gérontologique au Canada. La décennie de croissance sans précédent des années 1980 a été suivie d'une décennie caractérisée par des difficultés accrues et des perspectives réduites. À mesure que les ressources allouées à la recherche ont été réduites au cours des années 1990 et que le programme des politiques gouvernementales a changé consécutivement à l'addition de nouvelles exigences en matière de 
reddition de comptes et de pertinence, les chercheurs ont dû se tourner de plus en plus vers des solutions innovatrices afin de répondre à des questions de recherche critiques. D'une part, ceci a mené à une collaboration accrue entre les institutions, à la formation d'équipes de recherche multidisciplinaires et à l'établissement de relations et de nouveaux partenariats à l'extérieur du gouvernement pour le financement de la recherche. D'autre part, cette décennie de coupures dans les subventions a laissé la communauté des chercheurs canadiens avec une infrastructure de recherche inadéquate. $\Pi$ est bien connu que les investissements du Canada dans la recherche et le développement en proportion du produit national brut sont moindres que dans la plupart des autres pays industrialisés (Brochu, 1995). La création récente des Instituts de recherche sur la santé du Canada et, en particulier, d'un Institut du vieillissement, l'augmentation substantielle des subventions à la recherche associée à l'établissement des IRSC et le financement des Centres d'accès aux données de Statistique Canada et l'annonce du gouvernement fédéral sur l'augmentation du financement de la recherche (partie du programme électoral), tout cela augure une nouvelle ère pour la recherche gérontologique au Canada.

\section{Notes}

1 Les anniversaires sont également l'occasion de remercier ceux et celles qui ont apporté une aide incalculable au long du chemin. Mes fonctions de rédactrice en chef ont été grandement facilitées par le travail toujours consciencieux et complet de l'éditrice de $L a$ Revue, Rosemary Vanderkamp, assistee de Christa Parson. Je les remercie sincèrement toutes les deux. Louise Plouffe, de la Division du vieillissement et des aîné(e)s de Santé Canada, nous a offert son aide sous des formes très diverses, en vérifiant l'exactitude de la version française, en promouvant $L a$ Revue chaque fois qu'elle en avait l'occasion et en trouvant à plusieurs reprises les appuis nécessaires à nos initiatives. Sa défense de $L a$ Revue et le soutien qu'elle y a apporté ont permis la concrétisation de bien des idées. Je lui en suis immensément reconnaissante. Je remercie également Éric Jenkins, qui a apporté son aide aux traductions. La Revue ne pourrait pas fonctionner sans son Comité de rédaction, une équipe qui consacre bénévolement beaucoup d'heures à faire passer chaque manuscrit soumis par un processus exhaustif et rigoureux d'évaluation par des pairs. Depuis quatre ans, notre Comité de rédaction a eu pour membres John Carlson, Elaine Gallagher, Ellen Gee, Verena Haldemann, Réjean Hébert, Daphne Nahmiash, Sheila Neysmith, Joan Norris, Norm O'Rourke, Louise Plouffe, Karl Riabowol et Bernadette Ska. Je les remercie tous de leur engagement à notre revue et de tout le temps qu'ils lui ont accordé bénévolement. Mon rôle de rédactrice en chef a été facilité par le travail de mes prédécesseurs, Blossom Wigdor, Victor Marshall et François Béland. Blossom Wigdor et Victor Marshall "ont crée La Revue canadienne du vieillissement, l'ont développée et ont assuré son avenir" (Béland, 1991). François Béland a ajouté à l'envergure de La Revue en lui donnant un profil international et en renforçant sa réputation d'érudition et de rigueur. Pendant mes quatre années à la barre, le soin avec lequel mes prédécesseurs avaient établi la route à suivre m'a donné un avantage énorme.

2 Je remercie Joan Sims Gould de son aide dans la compilation de ces statistiques et des autres analyses comparatives du présent rapport. 


\section{Références}

Béland, F. (1991). The Canadian Journal on Aging enters its tenth year. Canadian Journal on Aging, 10(1), 4-5.

Béland, F. (1997). Building Canadian gerontology: A springboard for international recognition? Canadian Journal on Aging, 16(1), i-vi.

Brochu, M. (1995). Support for research on aging. Prepared for the Seniors Independence Research Program. Ottawa: Division of Aging and Seniors, Health Canada.

CARNET: The Canadian Aging Research Network. (1996). Into the age of aging: Selected findings, Toronto: Centre for Studies of Aging, University of Toronto.

Chappell, N.L., Joanette, Y., McDonald, L., Neysmith, S., Rockwood, K., Stones, M., \& Tuokko, H. (1999). Canadian Institutes of Health Research: The Canadian Institute on Aging/Institut canadien de vieillissement (Concept of operations). Victoria: Center on Aging, University of Victoria.

Connidis, I., Keating, N., Neysmith, S., Powell, C., Rockwood, K., \& Tierney, M. (2000). Beyond 1999: A research agenda. Canadian Journal on Aging, 19(3), $\mathrm{i}-\mathrm{v}$.

Gee, E.M. (1997). Policy and research on aging: Connections and conundrums. Canadian Journal on Aging and Canadian Public Policy-Analyse de Politiques, Special Joint Issue, CJA, 16(suppl.), i-viii.

Martin-Matthews, A. (2001). Gerontology in Canada: A decade of change. Contemporary Gerontology, 6(1): In press. 